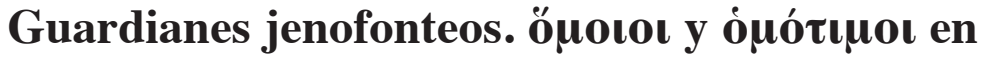 La constitución de los lacedemonios y Ciropedia
}

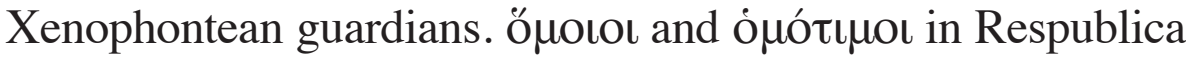 \\ Lacedaemoniorum and Cyropaedia
}

RODRIGO ILLARRAGA*

\begin{abstract}
Resumen: Acaso una de las marcas más notables del pensamiento político de los llamados filósofos socráticos haya sido la búsqueda por grupos sociales que, a cargo de gobernar, garanticen la estabilidad de una sociedad. Sin lugar a dudas los guardianes platónicos son la referencia paradigmática de este tópico, pero no la única; La constitución de los lacedemonios y Ciropedia tienen

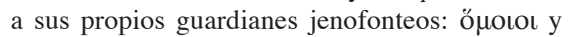

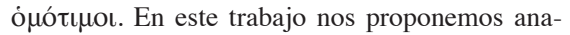
lizar estos dos grupos, proponiendo una lectura comparativa que dé cuenta de su relevancia para la filosofía política de Jenofonte.

Palabras clave: Jenofonte - Socráticos - Ciropedia - La constitución de los lacedemonios - Filosofía política.
\end{abstract}

\begin{abstract}
Perhaps one of the most remarkable features of the Socratic philosophers' political thought is the search for a group that, in charge of ruling, guarantee the stability of a society. the Platonic guardians are without doubt the paradigmatic reference of this topic, but not the only one. The Respublica Lacedaemoniorum and Cyropaedia have their own Xenophontean

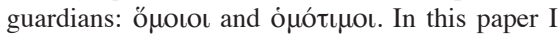
aim to analyze these groups, proposing a comparative reading that accounts for its relevance to Xenophon's political philosophy.

Palabras clave: Xenophon - Socratics - Cyropaedia - Respublica Lacedaemoniorum - Political philosophy.
\end{abstract}

Quizás uno de los signos destacables del pensamiento político del siglo IV a.C y, en particular, de las reflexiones sobre la política que se gestan en rededor del círculo de Sócrates, es la construcción de sistemas políticos de cuño irreal - como los que se proyectan de los vínculos entre la República de Platón y textos de la época como Asambleístas (Thesleff, 1995; Capra 2007a, 2010, Illarraga, 2016; Cf. Capra, 2007b, 2007c)-o bien la idealización de sistemas ya existentes - notablemente, el caso espartano (Cartledge, 1999) -, como modo de exponer en escenarios concretos los esquemas teóricos y de dinamizar el intercam-

Recibido: 03/03/2017. Aceptado: 16/12/2017.

* Universidad de Buenos Aires (UBA) y Consejo Nacional de Investigaciones Científicas y Técnicas (CONICET). Su investigación se centra en la ética y política de los filósofos socráticos, especiamente Jenofonte. Entre sus publicaciones recientes se cuenta "Enkráteia y gobierno. El gobernante insensato de Aristipo y su aparición en la Ciropedia de Jenofonte", Méthexis 30 (Brill), y la traducción del Hierón, Constitución de los lacedemonios, y Constitución de los atenienses (Jenofonte, Pseudo-Jenofonte), junto a C.Mársico y P. Marzocca (Buenos Aires: Prometeo-UNQui). Email: rodrigoillarraga@gmail.com. 
bio grupal en lo que se ha dado por llamar "zona de tensión dialógica” (Mársico, 2010). En los filósofos socráticos esta estrategia tuvo un lugar notable; no solo resaltan por ello el ya mencionado Platón y Antístenes, quien parece haber utilizado un recurso similar a la hora de analizar la sociedad ciclópea (Illarraga, 2012; Mársico, 2013), sino también Jenofonte. El filósofo, mercenario e historiógrafo ateniense construyó dos de sus obras de este modo, utilizando sociedades reales para, evadiendo la estricta fidelidad histórica, plantear parte de su propia filosofía política: Ciropedia, su magnum opus, es la semblanza de un gobernante ideal en base a la figura de Ciro, mientras que la breve Constitución de los lacedemonios, parte de las opera minora, retrata una sociedad ideal a partir de la Esparta organizada por Licurgo. Ambos textos son, junto con la restante obra de Jenofonte, unos de los exponentes paradigmáticos de un contexto de intenso debate sobre política (entendida en el sentido ámplio del término: cómo debe organizarse y gobernarse la sociedad) que se extendió por distintos géneros literarios: memorias, historiografía, encomios, descripciones de sistemas políticos, por solo mencionar algunos (Brown Ferrario, 2017, 57-60).

Tanto en la Ciropedia como en la Constitución de los lacedemonios se presenta un rasgo llamativo que, por su repetición, podemos pensar propio del pensamiento político jenofonteo: el retrato de una clase social sui generis, a cargo de buena parte de la administración

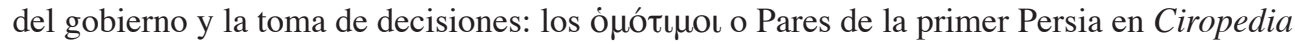
y los ónotor o Iguales lacedemonios de La constitución de los lacedemonios. A diferencia de la mayoría de los escasos estudios sobre estas cuestiones en Jenofonte, nuestro interés no radica en investigar cuánta verdad histórica hay en las descripciones jenofonteas (o las razones que habría tenido para alejarse de ella), sino ubicar las continuidades (y diferencias) entre las descripciones de los grupos mencionados: su lugar de garantía del funcionamiento del sistema político (que recuerda a los $\phi v ́ \lambda \alpha x \varepsilon \varsigma$ platónicos), los convierten en un elemento clave para la comprensión de la filosofía política de Jenofonte. De esta manera, nos dedicaremos a analizar cada uno de los grupos por separado, para luego realizar una comparación entre ambos.

Cabe señalar que la cuestión más amplia de la vinculación entre la presentación jenofontea de Esparta y Persia ha sido trabajada por estudios de principios de siglo XX. Wilhelm Prinz, en su introducción a De Xenophontis Cyri Institutione (1911) ha propuesto que Ciropedia debe ser leída como un gran panfleto en pos de la unión panhelénica contra el Imperio Persa, liga liderada por los espartanos, hipótesis recuperada por Bizos (1971). Jenofonte así encomiaría indirectamente a Esparta, a la vez que otorgaría claves y estrategias para la conquista de un imperio asiático, en un esquema en donde la Asiria que Ciro termina por conquistar representa a la Persia del siglo IV a.C. Esta propuesta, rápidamente criticada por Erwin Scharr (1919, 25-45), fue descartada para la lectura conjunta del problema PersiaEsparta (Cf. Tatum, 1989, 38-41). Conexiones menos extremas que la de Prinz han sido sostenidas por Luccioni (1949, 9, 32 y ss., 162), y Tigerstedt (1965-1978, 1: 159-179) y Georges también han abordado la cuestión $(1994,229)$. Más recientemente, y dentro de esta línea mesurada, Paul Christesen ha propuesto que la Ciropedia se configura como una propuesta de reforma militar que toca directamente a la estructura del ejército espartano (2006). Esn acaso el trabajo más completo de comparación entre la Persia de Ciropedia y las distintas presentaciones de Esparta en la obra de Jenofonte (Constitución de los lacedemonios, Agesilao), Christopher Tuplin (2002) se ha encargado de estudiar las conexiones que enlazan a las 
dos sociedades, en un esfuerzo por mostrar que no existe identidad entre ambas sociedades.

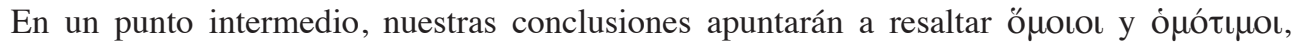
más allá de la estricta relación entre Esparta y Persia, denotan características claves para la filosofía política de Jenofonte.

\section{önoıoเ espartanos}

La constitución de los lacedemonios tiene un título engañoso: el gentilicio que aplica a

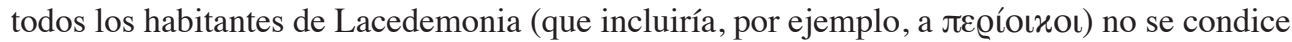
con lo tratado en obra, que atañe exclusivamente a los espartanos. En efecto, a los largo de sus quince capítulos, La constitución de los lacedemonios retrata la vida y normas de Esparta en un abanico de temas que comprende - si obviamos a fines organizativos el controvertido

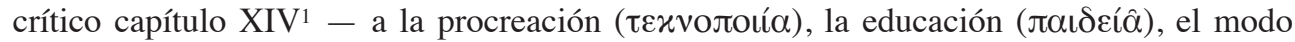

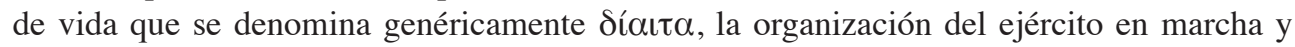
acampado, y finalmente la autoridad y las funciones del rey (Lipka, 2002, 44-46).

El grupo al que atañen todas las cuestiones relacionadas con la vida social, política y militar es el de los ö $\mu$ oıo: varones adultos que han atravesado las distintas instancias del sistema educativo espartano. Los ö $\mu$ oı oı son, en otras palabras, el resultado de este esquema pedagógico organizado por Licurgo que, para Jenofonte, es la razón que hace destacar a los espartanos por sobre todos los griegos ${ }^{2}$. Si seguimos la sugerencia de autores como Higgins $(1973,54)$ y Due $(1989,15)$ la educación no es, para Jenofonte, un momento o instancia puntual en la vida de los individuos, sino un constante interiorizar las normas y costumbres establecidas: ser un ǒmoıs s es estar atento a una constante revisión de la propia formación.

La peculiaridad de los ǒnoıo tiene su origen en la concepción, y quizás esa sea la razón por la que Jenofonte comienza, luego de una mínima introducción en donde aclara el origen de su interés, a desarrollar el tema de las técnicas para lograr niños fuertes y sanos (1.3-10), en sintonía con las teorías eugenésicas propias de su propio círculo intelectual - como las de Platón en República, entre otras (Cf. Roper, 1913; Lascarides, 2013, 4-6) - : solamente dos padres sanos, bien alimentados y ejercitados podrán gestar un hijo o hija con las mismas características (Blundell, 1995, 156-159).

$\mathrm{Ni}$ bien un niño espartano se encuentra en condiciones de entender lo que se le dice, la constitución espartana indica que debe ser puesto bajo las órdenes de los llamados $\pi \alpha \iota \delta o v o ́ \mu o \iota$, magistrados clave para la organización establecida por Licurgo con el poder

1 Existen varias posiciones sobre este debate. Un grupo sostiene que el capítulo XIV fue escrito posteriormente a los capítulos I-XIII, XV: Bazin (1885, 268 y ss.), Ollier (1934, xviii n.1), Delebecque (1957, 329-331), Luccioni (1947, 168 n.197) y, con alguna divergencia, Moore (1983, 72 y ss.). Wulff (1884, 44-49) y Chrimes (1948, 3 y ss.) sostienen una ordenación diferente, en donde el XIV es el primer capítulo. Marchant (1925, xxi y ss.), MacDowell $(1986,10)$ y Manes $(1988,19$ y ss.) proponen que tanto el XIV como el XV fueron escritos con posterioridad. Para un análisis completo de los argumentos en favor de una y otra posición, ver Lipka (2002, 28-31)

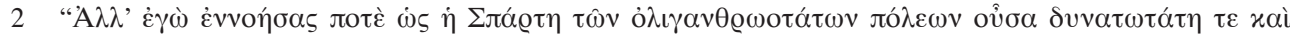

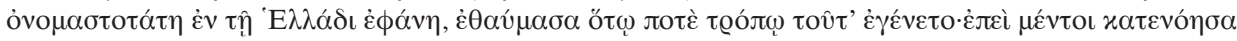

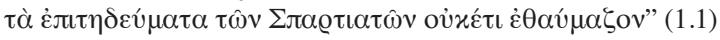

"En efecto, al comprender un día que Esparta, estando entre las ciudades con menos hombres, fue claramente la más poderosa y afamada en la Hélade, me admiré del modo en que llegó a suceder esto. No obstante, cuando estudié detenidamente las costumbres de los espartanos dejé de sorprenderme" 
para castigar a todos los que se encuentran bajo su control. El control de la educación por parte de funcionarios públicos es, de esta forma, una de las características distintivas de la

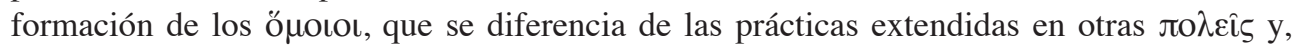
especialmente, en Atenas (2.1-2, Cf. Mársico, Illarraga y Marzocca, 2017, n. 192 y Moore, $175,99)$. Esta inspección rigurosa no recae exclusivamente en los oficiales establecidos, sino que existe un enérgico entramado que vuelca la potencia coercitiva en un número de instancias diferenciadas que se refuerzan en una dinámica retroalimentaria: jóvenes más avanzados en su educación tienen el privilegio de portar látigos para colaborar con el pedónomo castigando a quienes corresponda (2.1.), cualquier ciudadano adulto (esto es, cualquiera que haya atravesado el sistema educativo y, por lo tanto, sea parte de los ö dad para ordenar y castigar, y frente a la eventualidad de que no se encuentren pedónomos

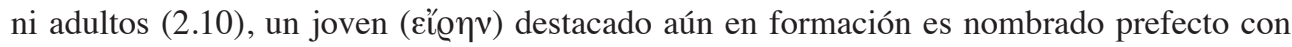
poder sobre los niños $(2.11)^{3}$. Al llegar a la adolescencia los controles se refuerzan, ya que es preciso lograr que en esa edad crítica, marcada por la arrogancia, los individuos se vuelvan en extremo modestos, característica que se espera los acompañe el resto de su vida ${ }^{4}$.

Al momento álgido de la juventud, el sistema educativo establece una situación agónica que se extenderá durante la vida de los ǒpotol. Los éforos, una de las máximas autoridades estatales del enrevesado sistema político espartano ${ }^{5}$, seleccionan de entre todos los jóvenes

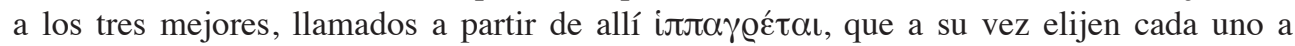
cien jóvenes más, en un proceso donde se deben hacer explícitas las razones por las que se escoge a alguien o se lo deja de lado (4.3-4). De esta forma, se establece una intensa y pública competencia, en donde se pone en evidencia el peso que tiene la mirada social sobre las conductas y reacciones de cada individuo. Esta dinámica, en donde el enfrentamiento es clave para intensificar las virtudes, se replica en el grupo organizado por los hipagretas. Estos trescientos, que son efectivamente los trescientos espartanos destacados para ser la guardia real y el cuerpo de elite del ejército lacedemonio, deben refrendar su posición constantemente; el conjunto seleccionado debe competir con aquellos que quedaron fuera de él, alentando la vigilancia y mejora permanente:

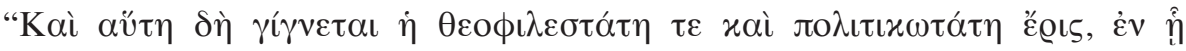

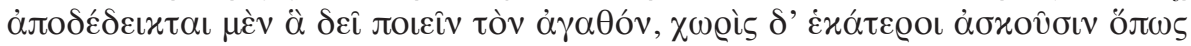

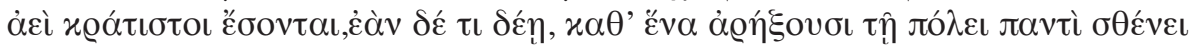
[äv]". (4.5)

3 Sobre los roles de las divisiones etarias en la educación, ver Chrimes (1952, 86-94), Ducat (2006, 97-99) y Kennell (2013, 381-387).

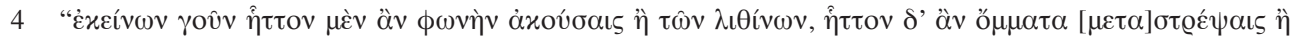

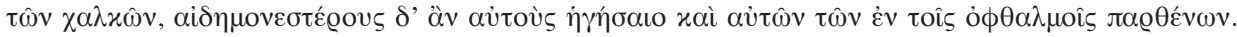

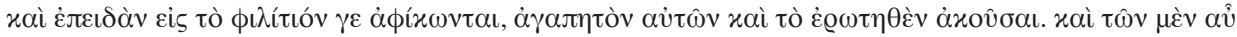

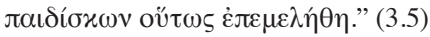

7 "Podrías escucharlos menos que a una estatua de piedra, menos intercambiarías miradas con ellos que con una figura de bronce y los creerías más pudorosos que la mirada de las doncellas. Cuando asisten a las comidas comunales, suerte si los escuchas responder. Así se ocupó de los adolescentes"

5 Sobre los éforos, ver Richer (1998, passim). 
"Sin duda estas competiciones llegan a ser las más gratas para los dioses y las más importantes para la ciudad, en las cuales se establece en verdad lo que debe hacer el hombre bueno, y cada uno de los grupos entrena por separado para ser el más fuerte, pero, si es necesario, los dos socorrerán con toda su fuerza a la ciudad"6

De esta forma, Jenofonte sintetiza lo que será una característica de la virtud de los ö́oı ı y, así, de la sociedad espartana: a la vez que la competencia pública alienta a mejorar el desempeño individual y permite establecer criterios observables por el conjunto de la sociedad, refuerza la estructura ciudadana general, en un modo que bien podríamos identificar como identitario. En otras palabras, las divisiones que tienen como fin un refuerzo de las virtudes sociales mediante enfrentamientos posibilitan también, por el establecimiento de valores generales, la unión frente a peligros externos.

Los ö $\mu$ oto en la legislación de Licurgo que expone Jenofonte, también se caracterizan por una relación particular con las gastronomía: ellas son públicas, para evitar que las normas referidas a la alimentación sean transgredidas (5.2), se realizan con comidas puestas en común (5.3), que deben ser modestas (5.3). Las comidas comunales refuerzan las reglas referidas al recato y moderación, y son realizadas en espacios compartidos, a fin de que el regreso al hogar fuerce a evitar excesos de vino y entrene en movimiento nocturno, útil en situaciones bélicas (5.6-7). De esta forma, todo lo relacionado con disposiciones gastronómicas, tocantes a en la dieta en sí misma y también a los modales de los comensales, tienen alcances que superan su ámbito inmediato: suponen un refuerzo de las conductas sociales tendentes a intensificar los valores establecidos por la educación brindada, a la vez que establecen y entrenan comportamientos útiles en otros ámbitos.

La comunalización observada en las comidas está extendida a todo aspecto que Jenofonte entiende como propietario:

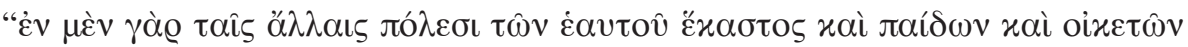

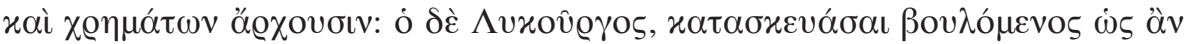

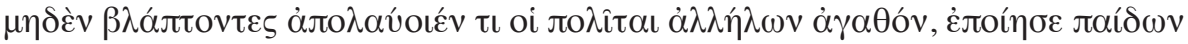

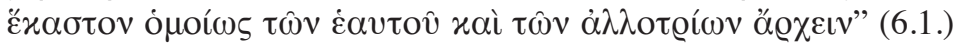

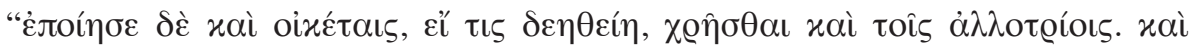

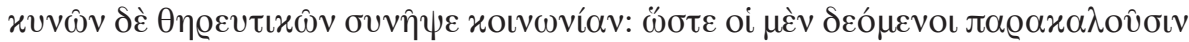

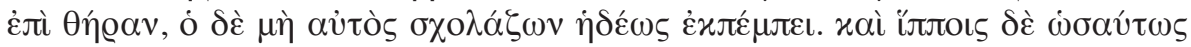

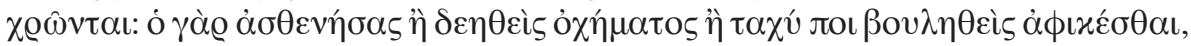

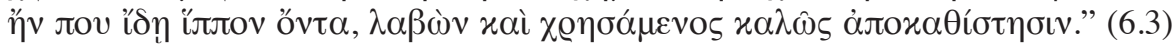

6 Las referencias a La constitución de los lacedemonios responden a la traducción de Mársico, Illarraga y Marzocca (2017), y a la traducción de Santsalvador (2007) para la Ciropedia. En ambos casos se han introducido variaciones, atentos a las ediciones de Dindorf (1888) y Marchant (1920). Asimismo, se han tenido en cuenta las traducciones y comentarios recientes. Para La constitución de los lacedemonios: Casevitz (2008) en francés, y Rico Gómez (1989) y Ramírez Vidal (2009) en español, de Moura (2002) en portugués, y Bowerscock (1968) y Gray (2007) en inglés. Para la Ciropedia: Bizos y Delebecque (1971-8) en francés, Ferrari (2013) en italiano, y Miller (1914), Marchant, Ambler (2011) en inglés. 


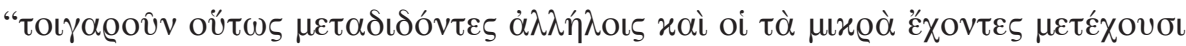

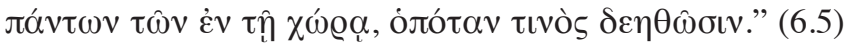

"En las otras ciudades cada uno manda sobre sus hijos, esclavos y bienes. En cambio, Licurgo, queriendo disponer que los ciudadanos hagan el bien el uno al otro sin perjudicarse en nada, estableció que cada uno mandase por igual sobre los propios hijos y sobre los ajenos"

“También hizo que si alguno lo necesitaba disponga de los esclavos domésticos de los otros. Asimismo, hizo comunes los perros de caza, de modo que quien los necesita para la caza los solicita, y el que no tiene el tiempo libre para cazar los manda con gusto. Y utilizan los caballos en la misma manera: quien está enfermo, necesita una cabalgadura o desea llegar rápidamente a algún lugar, si en algún lado ve que hay un caballo, tras tomarlo y usarlo correctamente, lo devuelve."

"Por lo tanto, de este modo, al compartir los unos con los otros, los que tienen poco participan de todos los frutos de la tierra cuando sea que alguno lo necesita."

Hijos, esclavos, y bienes materiales quedan sujetos a las necesidades de cada uno de los individuos, logrando así articular las demandas del conjunto social y evitando diferencias que desintegren el fuerte tejido coercitivo (Cf. Mársico, Illarraga, Marzocca, 2017; Illarraga, 2017). Acompañando estos preceptos relacionados con los usos comunes, Jenofonte reseña que Licurgo estableció leyes que impiden las actividades pecuniarias (7.1-2): además de no existir incentivo alguno para acumular riquezas (ya que todos los bienes son compartidos), el establecimiento de una moneda de compleja atesoración (7.5) y una serie de castigos y requisas a quienes tienen oro y plata (7.6) completan un panorama que anula los peligros que la diferenciación por enriquecimiento podría generar en la estructura comunal de los ǒnotot.

Es de notar que el esquema propuesto para los ö $\mu$ oเo no está circunscripto a los primeros grupos etarios, sino que se extiende a lo largo de la vida de los individuos. Como hemos señalado, a lo largo de distintas formas e instancias, se aplican diferentes incentivos pedagógicos para orientar la formación anímica de cada uno de los ǒ de esta política se encuentra en la legislación que se presenta para evitar el descuido de la virtud durante la vejez (10.1). En esta etapa es cuando los $\gamma \dot{\varepsilon} \varrho o v \tau \varepsilon \varsigma$ pueden acceder a uno de los más importantes órganos de toma de decisiones, la Gerusía. La posibilidad de formar parte de una instancia que tiene autoridad sobre procesos capitales (10.2) impulsa a aquellos que se encuentran al final de sus vidas a mantener los modos aprendidos previamente. A la vez, permite poner en función de la sociedad todo un extenso acervo de experiencia y conocimiento.

Resumamos entonces las principales características de las normativas que constituyen a los ö $\mu$ oto en un grupo distintivo:

(a) Educación a lo largo de la vida — incluida la vejez —, a cargo de funcionarios públicos, en señaladas etapas $(2,10)$. 
(b) Especial atención a la mirada social como forma coercitiva, en donde la sociedad refuerza los valores impartidos pedagógicamente $(2.2,3.2-5,4.5-7,4.4-6,7.3,8.1$, 9-4.6, 10.4-6).

(c) Pudor y modestia como virtudes fundamentales (3.5.2-3).

(d) Competencias intrageneracionales para alentar la formación y el progreso (4).

(e) Regulaciones gastronómicas con objetivos sociales: comidas comunes y restricciones dietarias (5).

(f) Comunalización de bienes (6).

(g) Prohibición de trabajo pecuniario y de acumulación de oro y plata (7).

(h Grupo de los ancianos a cargo de los más importantes órganos jurídicos (10.2).

\section{ónótıนot persas}

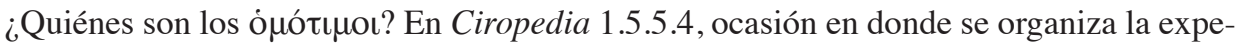
dición de apoyo militar a Media que da inicio a la peripecia central de la obra, aparecen por primera vez nombrados como tales aquellos persas que participan del gobierno y del cuerpo principal del ejército (son, en rigor, la infantería de línea con formación de élite, acompañada por los soldados del pueblo llano: honderos, arqueros y peltastas).

No obstante, es en la sección que se extiende desde 1.2.1 hasta 1.2.15 donde Jenofonte aborda a esta clase a partir de su proceso de formación, anotando de esta forma las prácticas, valores y modos de vida asociada a ella. Para ser un ó ó$_{\tau} \iota \mu \mathrm{s}$ no existe mayor condición que la de haber atravesado esta educación — extendida a lo largo de toda la vida - , abierta teóricamente a todos los persas:

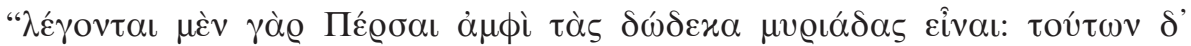

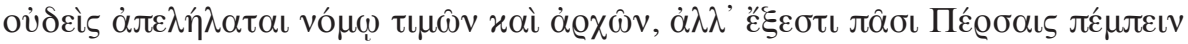

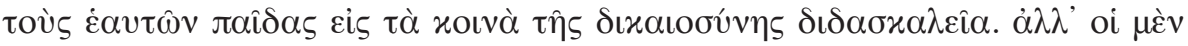

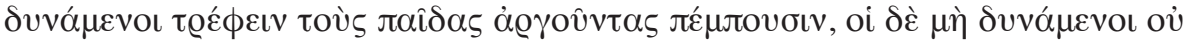

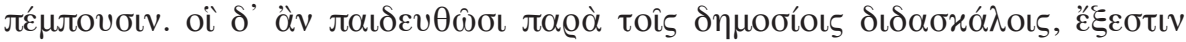

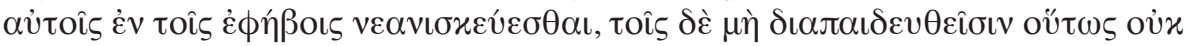

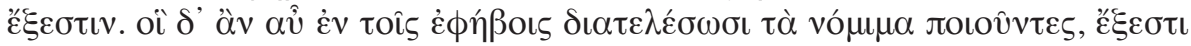

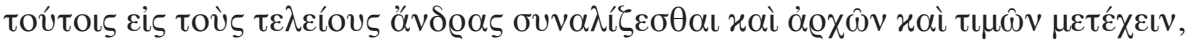

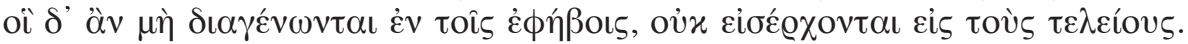

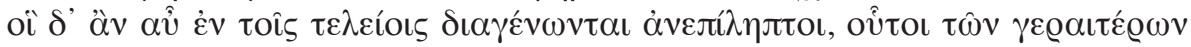

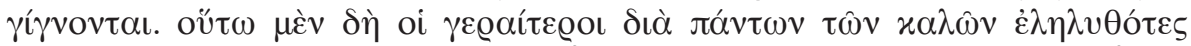

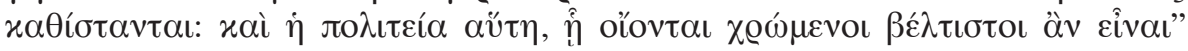
$(1.2 .15 .3-18)$

"Se dice que los persas son cerca ciento veinte mil, y ninguno de ellos es apartado por ley de honores o cargos públicos, sino que todos los persas tienen derecho a enviar a sus hijos a las escuelas públicas de justicia. Pero sólo los que pueden educar a sus hijos sin hacerles trabajar los envían allí, y los que no pueden no los envían. Los niños que hayan sido educados con maestros públicos están permitidos a pasar su juventud en la clase de los efebos, mientras que los que no han recibido esa educación no 
tienen permiso. Por su parte, los que hayan cumplido las normas vigentes en la clase de los efebos tienen el derecho de sumarse a la clase de los adultos, y de participar en cargos y honores; en cambio, los que no hayan vivido en la clase de los efebos, no tienen acceso a la de los adultos. A su vez, los que vivan intachablemente en la clase de los adultos pasan a formar parte de la clase de los ancianos. Así, la clase de los ancianos está compuesta de aquellos que hayan pasado por todos los niveles de virtud. Y ésta es la constitución con cuya práctica creen que pueden convertirse en los mejores."

El acceso al grupo gobernante se posibilita a partir del tránsito pautado a través de un riguroso y prolongado cursus honorum que supone, a cada paso, la posibilidad de ser eliminado frente a la eventualidad de no cumplir las normativas y expectativas dispuestas. Sólo al alcanzar el estatus de adulto se pueden ocupar cargos públicos y llegar a este momento significa haber recorrido el dilatado pasaje por el grupo de niños y efebos. Si bien no existe prescripción legal que impida emprender el curso pedagógico persa, la entrada al primero de estos grupos está supeditada a la realidad de cada grupo familiar: quien tenga la capacidad económica como para prescindir de sus hijos podrá enviarlos a las escuelas públicas de justicia. Dado que Jenofonte caracteriza a Persia como una sociedad pequeña y rural, esta mención no es ingenua ni desdeñable: en una economía agraria antigua es fundamental contar con la mano de obra suplementaria provista por el grupo familiar, con lo que la participación y permanencia en el sistema educativo público queda reducida al estrecho sector social con amplios recursos económicos (Cf. Due, 1989, 207-215; Nadon, 2001 26-41; Whidden, 2007, 542 y ss.; Danzig, 2012, 501). A partir de esto, es de suponer la reproducción de la división entre estos dos estamentos (a saber, los que pueden acceder a la educación y por ello participar del gobierno, y los que no) de forma bastante lineal: los sectores pudientes son formados de acuerdo a los criterios establecidos y por ello acceden al poder, los honores y las riquezas, lo que a su vez permite que las próximas generaciones se aseguren su lugar en el sistema.

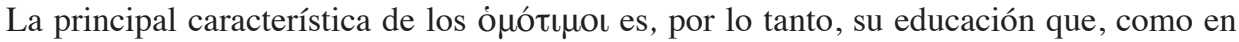
el caso espartano, no consiste en un momento específico relegado a las primeras décadas de existencia, sino por un conjunto de enseñanzas y, más importante, de prácticas extendidas durante el transcurso de la vida. Toda ella, para aquellos que entran en el sistema público, transcurre en una óyo@ó rodeada de edificios en donde cada uno de los grupos

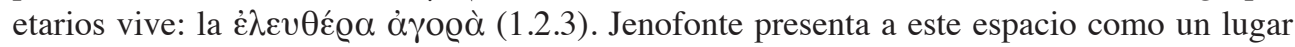

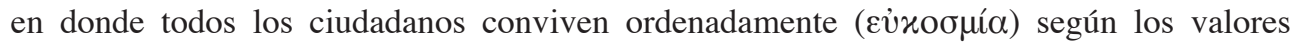
persas, en donde los comerciantes se encuentran vedados por su desorden ( $\tau \dot{\varrho} \varrho \beta \eta)$ y malas prácticas (Cf. Whidden, 2007, 543). Esta disposición, que comprende también la vivienda comunal, es una garantía del bien común: la mutua vigilancia tácita que se establece en la áyo@ó fuerza a los ciudadanos a sostener los valores aprendidos a lo largo de la educación (Illarraga, 2017, 89-90).

Cada uno de los estamentos etarios por los que deben pasar los ó $\mu$ ó $\iota \mu$ o está caracterizado por un conjunto de reglas que, si bien se encuentra armonizado con el sistema en general, tiene aspectos específicos para cada grupo. La clase de los niños ( $\pi \alpha \hat{\imath} \delta \varepsilon \varsigma$ ) es formada con atención en lo anímico: mediante una suerte de juicios diarios, los niños son entrenados 
en la justicia. Como muestra la intensamente debatida anécdota de los niños y sus túnicas de 1.3.16-18, la concepción de justicia para los persas tiene un carácter estrictamente jurídico: justo es aquello que se ajuste a las normas establecidas, siendo irrelevante si ellas atentan directamente contra el bien común o el mejor ordenamiento posible (Cf. Luccioni, 1947, 65; Newell, 1983; Gera, 1993, 74-78; Ferrari, 1995, 113 n. 43; Nadon, 2001, 47-49; Danzig, 2009, 243). Este atenerse a la ley se condice también con otra baza educativa persa: la obe-

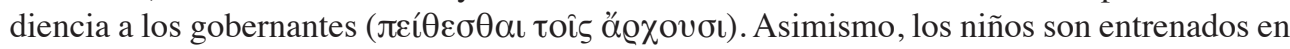

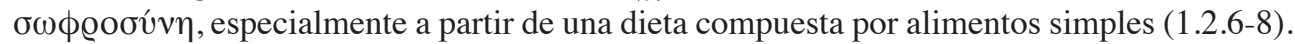

Los efebos ( $(๕ \phi \eta \beta O \iota)$, por su parte, centran su formación en el entrenamiento físico, brindado en este caso mediante servicios realizados a petición de las autoridades, y de oficiar de la guardia del rey en partidas de monteo. En estas salidas cinegéticas los jóvenes entrenan con algunas de las armas que luego, al convertirse en adultos, usarán en las actividades militares, de modo que la caza es entendida como propedéutica para la guerra. En esta línea, se llevan adelante prácticas (soportar el frio y el calor, ejercitar la marcha, atacar a fieras, etc.) a fin de acumular experiencia para futuros enfrentamientos. También se encuentra presente en énfasis en la mesura: fungir de guardia real con alimentos mínimos tiene como fin endurecer a los efebos y alentar que valoren sus ya ascéticas comidas. Finalmente, en esta etapa se llevan adelante enfrentamientos populares ( $\eta \eta \mu$ ó เo $\dot{\alpha} \gamma \omega \hat{v} \varepsilon \varsigma$ ) en donde se

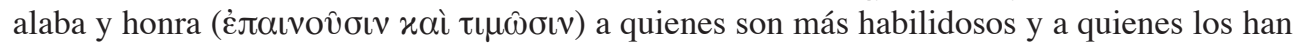
entrenado, de modo que la virtud es alentada públicamente (1.2.9-12).

La próxima etapa, para aquel efebo que se atiene a los valores enseñados, es la clase

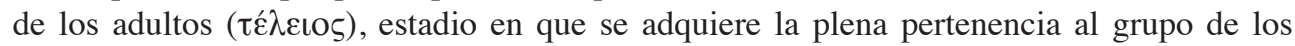

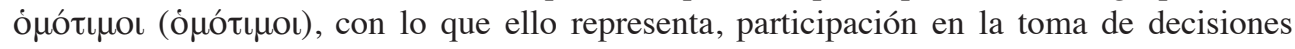
gubernamentales y en la guerra:

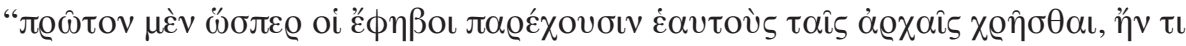

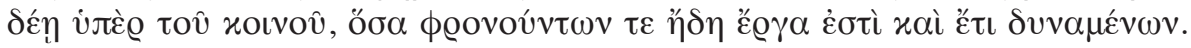

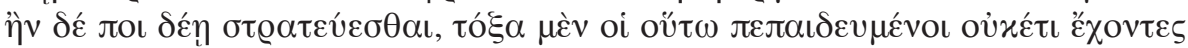

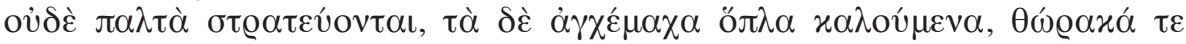

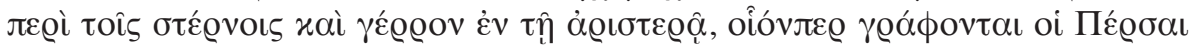

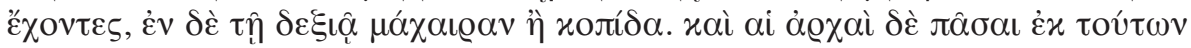

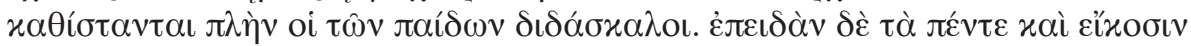

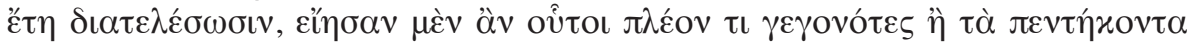

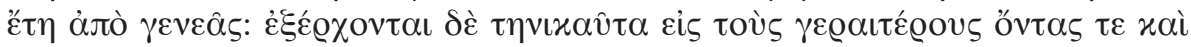

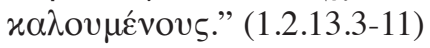

"Se ponen al servicio de las autoridades, por si hubiera que realizar cualquiera de las acciones relativas a la comunidad que son ya propias de personas sensatas pero aún vigorosas. Si se ha de ir de expedición, los que han sido así educados marchan sin ni siquiera flechas ni lanzas, sino con las llamadas armas de cuerpo a cuerpo: una coraza alrededor del pecho, un escudo en la mano izquierda, como llevan los persas en las pinturas, y en la derecha una daga o un cuchillo. Es de esta clase de donde son designados todos los cargos públicos, excepto los maestros de los niños” 
Alrededor de los cincuenta años de edad, los miembros de la clase adulta se convierten en parte del estrato de los ancianos ( $\gamma \varepsilon \varrho \alpha i ́ \tau \varepsilon \varrho o l)$. En este grupo, que está compuesto «por

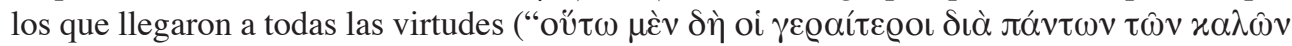

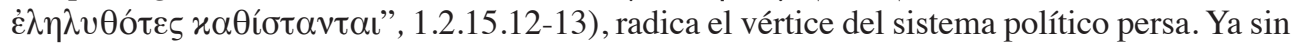
participar de la vida castrense, los ancianos se ocupan de juzgar todos los asuntos públicos y

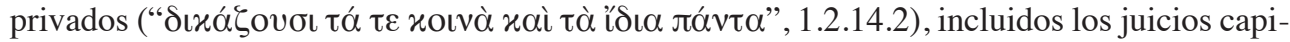
tales y seleccionan, de entre los adultos, a quienes ocuparán los distintos cargos de gobierno

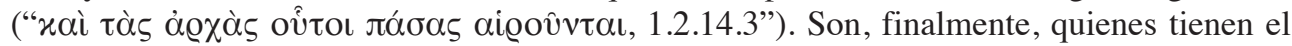
derecho a expulsar a quienes, a lo largo de su vida, han mostrado falencias en su formación.

Como han señalado varios autores, esta descripción de los ó libro I atañe hasta el momento de comenzar Ciro las campañas que darán inicio al Imperio Persa (Nadon, 2001, 61 y ss.; Danzig, 2009; Johnson, 2005). Ya de cara a la organización de la contienda con Media en 2.1.9, se comienza a estructurar un nuevo esquema social, que tendrá como característica distintiva la inclusión de persas "comunes" en el cuerpo de élite de los ónótıuo a partir de la distribución del mismo armamento para todos. De esta forma, el conjunto de soldados no educados bajo el sistema de escuelas públicas (relegados hasta aquí a posiciones auxiliares durante conflictos bélicos) se integra al aparato militar plenamente, logrando de esta forma acceder a un modo de ascenso social no marcado por las posibilidades económicas que eran la clave de acceso a la educación persa. Si bien el grupo de los ónótıนo sigue existiendo, tal y como es descripto en el libro I, es evidente que, al final de la Ciropedia, ya no son el núcleo en torno al que orbita la sociedad imperial, sino que forman parte de un entramado mayor.

Sintetizando lo revisado, las características de los ó $\mu$ ó $\iota \mu$ o pueden ser sintetizadas de la siguiente manera:

(a) Educación extendida a lo largo de la vida, distinguida por diferentes etapas (1.2.15).

(b) Especial atención a la mirada pública como elemento coercitivo que refuerza la

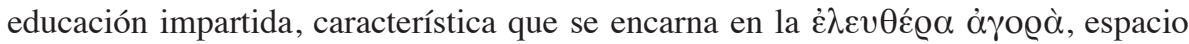
donde transcurre la vida de los ónótıнo (1.2.3).

(c) Mesura como valor fundamental (1.2.8).

(d) Competencia como aliento del progreso (1.2.12).

(e) Elementos dietarios como refuerzo educativo $(1.2 .6,1.2 .11)$.

(f) Comunalización de viviendas y tareas $(1.2 .3,1.2 .9,1.2 .13)$.

(g) Crítica al trabajo pecuniario e imposibilidad de realizarlo (1.2.3).

(h) Clase de los ancianos a cargo de los más importantes órganos judiciales y del nombramiento de oficiales públicos (1.2.14-15).

\section{Una comparación entre persas y espartanos}

(a) Lac.: Educación a lo largo de la vida -incluida la vejez -, a cargo de funcionarios públicos, en señaladas etapas $(2,10)$. // Cyr.: Educación extendida a lo largo de la vida, distinguida por diferentes etapas (1.2.15). 
La noción de educación no como etapa concreta reducida a un momento determinado de la vida (Higgins, 1973, 54; Due, 1989, 15; Lu, 2015, part 2), sino que debe ser extendida a

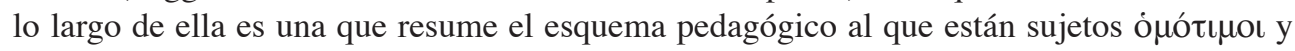

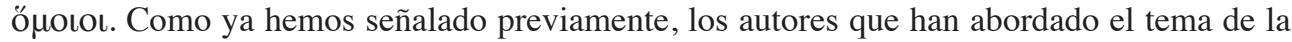
$\pi \alpha \iota \delta \varepsilon\{\hat{\alpha}$ en Jenofonte en estos casos no han dudado en señalar que este sistema educativo apunta a reforzar durante diferentes instancias los valores y formas estimados como favorables para la sociedad. De esta forma, la educación se entiende, antes que en la adquisición de cierto acervo de conocimiento, como la interiorización de una ética del bien común. En los casos persa y lacedemonio existe una división por grupos etarios, no idénticos pero sí semejantes. Tuplin $(2002,153)$ ha realizado un cuadro comparativo para describir esta situación ${ }^{7}$ :

\begin{tabular}{|c|c|}
\hline Persia & Esparta \\
\hline 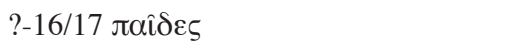 & (7-18/9) $\pi \alpha i ̂ \delta \varepsilon \varsigma$ \\
\hline 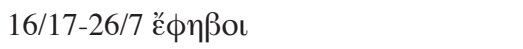 & 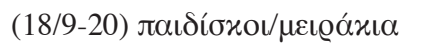 \\
\hline 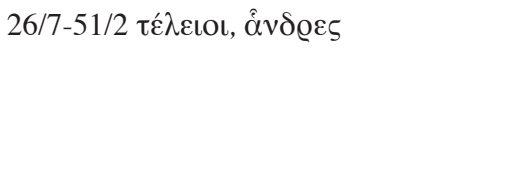 & $\begin{array}{l}\text { (20-30) } \dot{\eta} \beta \hat{\omega} v \tau \varepsilon \varsigma \\
\text { (30-?) hombres más allá de su } \\
\text { juventud,ocupantes de posiciones } \\
\text { gubernamentales }\end{array}$ \\
\hline $\begin{array}{l}\text { 51/2- hombres por sobre la edad militar, } \\
\gamma \varepsilon \varrho \alpha i ́ \tau \varepsilon \varrho o l\end{array}$ & 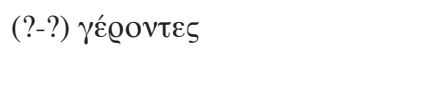 \\
\hline
\end{tabular}

Es de señalar que tanto en Persia como en Esparta el esquema de educación extendida se desarrolla en forma diferente de acuerdo al grupo etario. Los grupos iniciales (las primeras dos clases para el caso persa, las primeras tres para el lacedemonio) tienen una educación formal a cargo de funcionarios públicos, mientras que los grupos adultos y ancianos no cuentan con ella. Si en los primeros casos existen pedagogos asignados por los magistrados con la función de brindar conocimientos concretos pero también de orientar a grandes rasgos el modo de conducta propia del ciudadano, en los últimos la educación (entendida en sentido laxo) se realiza mediante elementos indirectos.

(b) Lac.: Especial atención a la mirada social como forma coercitiva, en donde la sociedad refuerza los valores impartidos pedagógicamente (2.2, 3.2-5, 4.5-7, 4.4-6, 7.3, 8.1, 9-4.6, 10.4-6). //Cyr.: Especial atención a la mirada pública como elemento coercitivo que

7 El original, con transliteraciones. 


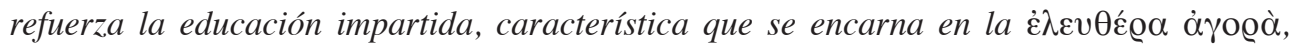

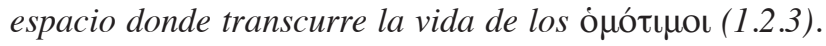

La pedagogía indirecta no es otra cosa que un complejo de distintas instancias de control y coerción social, mecanismos tendentes a disciplinar a los individuos. Esto es abiertamente señalado por Jenofonte como una diferencia con otros estados tanto en la Ciropedia como en la Constitución de los lacedemonios, donde se explicita que las leyes de Persia y Lacedemonia, antes que ocuparse de establecer castigos para quienes las contravienen, se preocupan por compeler a un actuar virtuoso. Si bien la función pedagógica de la legislación no olvida un carácter punitivo, el objetivo principal de la ley es formar el carácter del individuo para evitar llegar al incumplimiento y la sanción correspondiente:

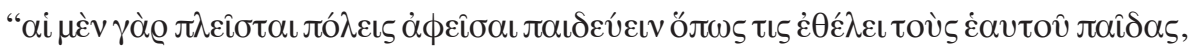

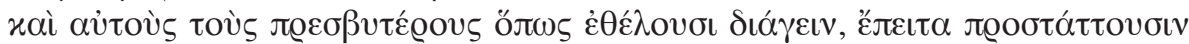

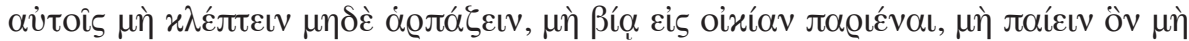

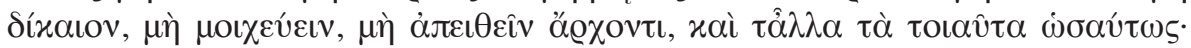

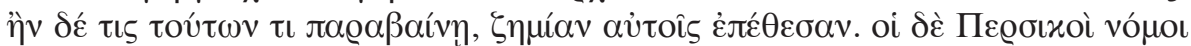

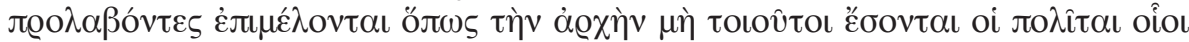

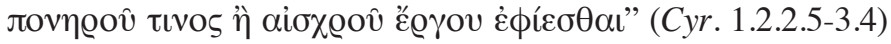

"Pues en la mayoría de las ciudades, dejando que cada cual eduque a sus hijos como deseen, luego les ordenan no robar ni saquear, no irrumpir en ninguna casa con violencia, no golpear a quien no sea lícito, no cometer adulterio, no desobedecer al gobernante y, asimismo, cosas por el estilo; y si alguien infringe uno de estos preceptos, le imponen un castigo. En cambio, las leyes persas se anticipan preocupándose de que, desde el principio, los ciudadanos no sean tales que tiendan a alguna acción ruin o vergonzosa"

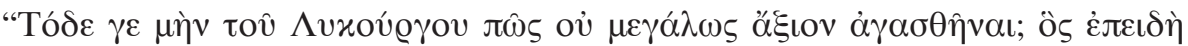

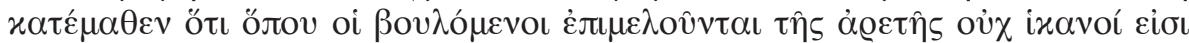

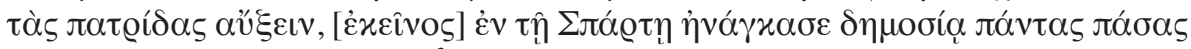

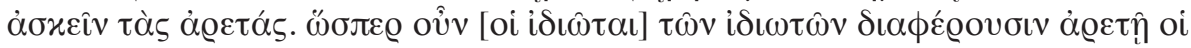

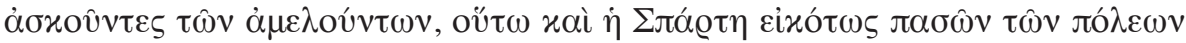

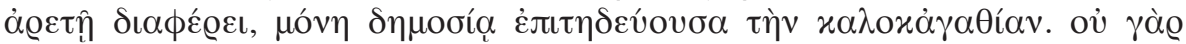

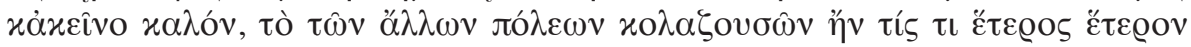

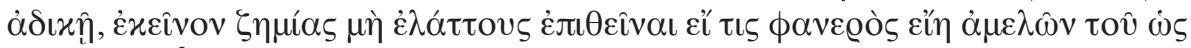

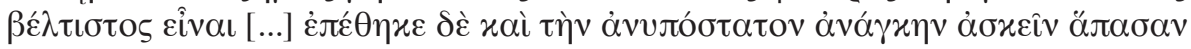

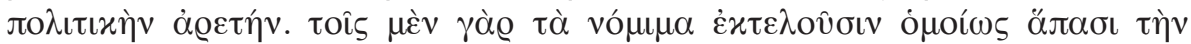

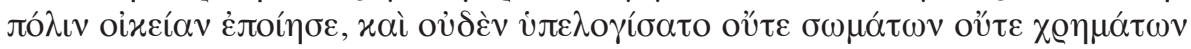

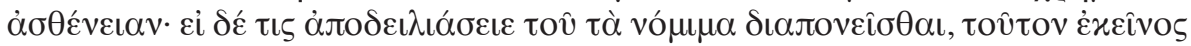

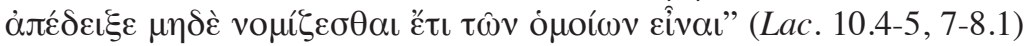

“¿Cómo esta idea de Licurgo no va a ser enormemente digna de admiración? Cuando entendió que donde se preocupan de la virtud los que quieren no son suficientes para engrandecer su patria, en Esparta obligó a todos a practicar todas las virtudes públi- 
camente. Entonces, como los individuos se diferencian unos de otros por la virtud, ya sea que practiquen o la olviden, así también del mismo modo se diferencia Esparta de todas las otras ciudades en cuanto a virtud, por ser la única que persigue la virtud públicamente ¿No es hermoso que, mientras las otras ciudades castigan si alguien hace daño en algo uno al otro, él impulsó castigos no menores a quien abiertamente se despreocupa de ser lo mejor posible? [...] Además, impuso la ineludible necesidad de practicar la virtud política integral, pues para los que cumplen todo lo establecido hizo a la ciudad propia para todos con iguales derechos y no tomó en cuenta la debilidad ni de sus cuerpos ni de sus bienes: si alguno flaqueó sirviendo a la ley, a ese le quitó incluso el ser considerado entre los iguales."

En los dos textos, la pertenencia al grupo ciudadano está atravesada de la obligación por atenerse a un esquema de obligaciones de amplio espectro: independientemente de una normatividad minuciosa en ciertos detalles, el eje del sistema es una ética centrada en la virtud ciudadana. Esta virtud sólo puede existir, según lo expuesto por Jenofonte, a partir de una decisión (tomada por Licurgo en el caso espartano, y atribuida a la tradición en el persa) que reorganice la sociedad de forma radicalmente diferente a la conocida en el mundo griego. Para lograr el objetivo propuesto, tanto ö comunitarios que refuerzan la educación impartida: son ellos mismos en tanto grupo los encargados de revisar constantemente la conducta de sus pares.

(c) Lac.: Pudor y modestia como virtudes fundamentales (3.5.2-3) // Cyr.: Mesura como valor fundamental (1.2.8).

Tuplin ha señalado, con respecto a las conexiones entre estas características anímicas, que existe un lazo tenue $(2002,154)$. Efectivamente, el autor anota la importancia de la modestia (aỉós) para el caso de los jóvenes espartanos y la compara con la actitud de Ciro - recordemos que él pertenece a los ónótıuol- en su adolescencia (1.4.4), pero señala que hasta allí llegan las similitudes. Tras ello, realiza un detallado análisis de las virtudes enseñadas en cada etapa, mostrando las diferencias puntuales entre ambos sistemas (2002, 155-161). Si bien no podemos más que acordar con su completo y ajustado estudio, creemos que es posible - aun aceptando las distancias y divergencias por él señaladascentrar la mirada en las continuidades existentes. Due ha marcado el espectro amplio del

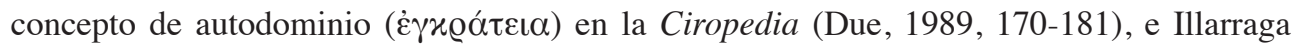
(2017, 91 y ss.) han propuesto cómo este autocontrol se vincula con otros puntos clave de la educación persa, como la gratitud. En esa línea, consideramos que efectivamente existe un gran campo semántico que engloba a nociones como la de aió́s (para el caso

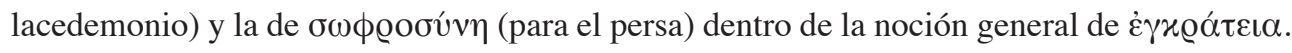

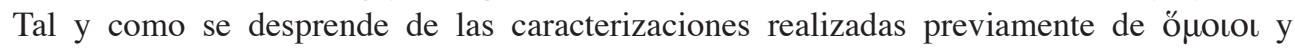
ó refrenen ciertas actitudes y comportamientos. Sería posible argumentar la trivialidad de esta afirmación marcando que toda sociedad se construye a partir de este autodominio, frente a lo

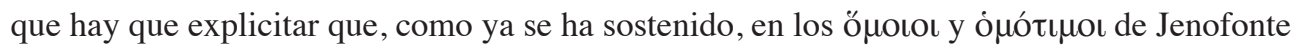


la prioridad está en la constitución del carácter anímico y no en el autodominio ejercido en determinada situación de cara a la evaluación de un castigo futuro ${ }^{8}$.

(d) Lac.: Competencias intrageneracionales para alentar la formación y el progreso (4). II Cyr.: Competencia como aliento del progreso (1.2.12).

Otra de las características compartidas es la competencia intragrupal como forma de incentivar y celebrar las virtudes. En el caso de Esparta, este $\alpha \hat{\gamma} \omega \dot{v} v$ tiene un momento paradigmático: la selección de los hipagretas y de los trescientos, y los enfrentamientos que se realizan entre ellos y los no seleccionados a fin de revalidar el honor dado. Durante la etapa correlativa persa, los epheboi también prueban su valía en enfrentamientos populares. Este esquema, que alienta al individuo a mostrar al máximo sus capacidades, no se contradice con el lugar dado a la $\dot{\gamma} \gamma \varkappa \varrho \alpha ́ \tau \varepsilon \iota \alpha$, virtud ceñida al ámbito que vincula al individuo con las normas sociales y las autoridades. En otras palabras, el autodominio no debe ser entendido como humidad o mesura para con la exposición de las propias habilidades. Esto, que podría ser visto como una contradicción, tiene sus similitudes, mutatis mutandis, con el esquema anímico que Sandridge ha propuesto para la $\phi \iota \lambda \alpha v \theta \varrho \omega \pi i ́ \alpha$ de Ciro, donde la preocupación por los otros no invalida la persecución del propio interés (2012, 33-42, 63-64, 93-94).

(e) Lac.: Regulaciones gastronómicas con objetivos sociales: comidas comunes y restricciones dietarias (5). I/ Cyr.: Elementos dietarios como refuerzo educativo (1.2.6, 1.2.11).

Este punto, quitando las minucias que se precisan en La constitución de los lacedemonios y las restricciones a la bebida que allí aparecen, es de plenas coincidencias. Las dietas

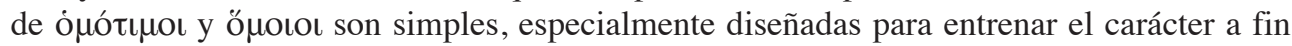
de soportar la carestía, práctica especialmente útil para tiempos de guerra. Si bien no se lo especifica en Ciropedia, es de suponer la práctica de comidas comunes en las viviendas

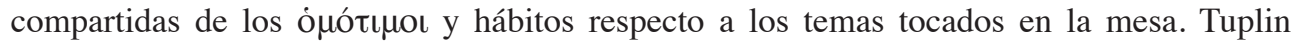
ha señalado con razón que las comidas de Ciro durante su campaña comprenden temas inapropiados para los estándares presentes en La constitución de los lacedemonios (2002, 143-144), pero como hemos marcado, allí ya no estamos en presencia del relato estricto de la vida de los ónótıนo, sino frente a un nuevo modo de convivencia inaugurado por el príncipe persa. Si, en cambio, recurrimos a la primera impresión que tiene Ciro de niño

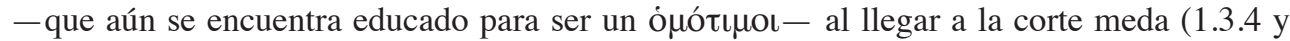
ss.), es fácil observar el reproche que se hace a la bebida en exceso y la liberalidad con la que se comportan los comensales. Tenemos así una coincidencia de prácticas que devela el carácter doble de las regulaciones dietarias: por un lado, la selección de los alimentos y sus cantidades sirve como adelanto de las posibles vivencias bélicas, mientras que las disposiciones más generales (tópicos de conversación, comidas compartidas, cantidad de bebida) sirven como refuerzo de las conductas deseables en todo momento.

(f) Lac.: Comunalización de bienes (6). // Cyr.: Comunalización de viviendas y tareas $(1.2 .3,1.2 .9,1.2 .13)$.

8 Esta apreciación es válida aún en el caso del robo de alimentos de Lac.2.7-10: nunca es celebrada la incontinencia, sino la capacidad y destreza necesaria para robar algo sin ser descubierto. 
La comunalización de bienes de los ö llamativos para la tradición y acaso es el punto de mayor divergencia con los ó persas. En Esparta la condición de ö $\mu$ oıo es otorgada por defecto a todos los nacidos de öhoı que prueben ser dignos de ella, y la igual distribución de propiedades y riquezas garantiza la marca de los iguales: que no haya diferencia alguna entre ellos, más allá de las mínimas que mediante el mérito se puedan adquirir (en particular, el acceso a la Gerusía).

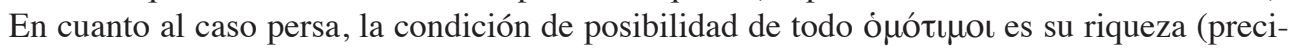
samente aquello que los separa del pueblo que no puede acceder a la educación pública), y es de suponer que existen diferencias entre las haciendas de unos y otros. No obstante, las fortunas no tienen injerencia política una vez accedido a las escuelas comunitarias: a partir de allí, los grados de responsabilidad que se adquieran (en particular, alcanzada la clase de los adultos) dependerán de la habilidad y virtud de cada individuo.

(g) Lac.: Prohibición de trabajo pecuniario y de acumulación de oro y plata (7). // Cyr.: Crítica al trabajo pecuniario e imposibilidad de realizarlo (1.2.3).

A la luz del anterior punto cobra plena dimensión la prohibición de realizar un trabajo que genere ingresos y acumular oro y plata: más allá de la razón declarada — solapación del entrenamiento guerrero - , válida para el sistema lacedemonio, la persecución de ejercicios lucrativos traería aparejada la posibilidad de acumulación de riquezas, que demolería el axioma comunalizador espartano. El caso persa es diferente. Las fortunas ó $\mu$ ótıนo obligan a Jenofonte a producir un sistema coherente, con lo que este aspecto replica un sistema aristocrático tradicional: se rechazan las características adscriptas al trabajo y se estipula una agenda centrada en lo militar y gubernamental que excluye implícitamente a la labor manual.

(h) Lac.: Grupo de los ancianos a cargo de los más importantes órganos jurídicos (10.2). // Cyr.: Clase de los ancianos a cargo de los más importantes órganos judiciales y del nombramiento de oficiales públicos (1.2.14-15).

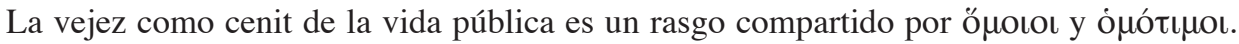
Este punto álgido no se obtiene por la mera acumulación de años de vida, sino que depende de una ajustada obediencia a los códigos aprendidos, mostrando así un aspecto de la crítica habitual en los socráticos a los no-especialistas ${ }^{9}$. Los ancianos acceden a las posiciones más sensibles en el gobierno precisamente porque son ellos quienes pueden dar cuenta de una formación completa y acabada en la que se han mostrado probos. En ellos se depositan las funciones clave para el correcto devenir de la sociedad: el nombramiento de los magistrados u oficiales públicos (notablemente, los funcionarios encargados de la formación pedagógica de los niños) y de los juicios capitales.

\section{Conclusión}

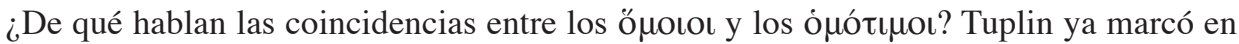
su investigación sobre los vínculos generales de Esparta y Persia que "no hay una forma razonable en la que se pueda decir que la Ciropedia es un libro sobre Esparta en la que

9 e.g., Antístenes en $F S 849$ (= SSR, V.A. 84) y ss. 
Persia es usado como un mero disfraz" y que "tenemos pocos argumentos para atribuirle a Jenofonte la formulación de su ideal en Ciropedia entendiéndolo efectivamente asentado en Esparta" $(2002,162)$. Aun atendiendo a esta idea, es preciso señalar que los vínculos entre las clases gobernantes de las dos sociedades retratadas, los ö $\mu$ oı

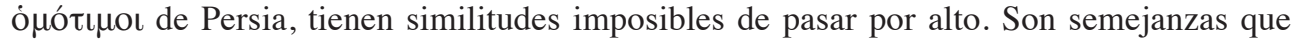
no implican identidad, ya que existen diferencias que hacen imposible sostener que uno y otro grupo son los mismos con diferentes ropajes, pero sugieren la importancia de una lectura conjunta. La primera de nuestras conclusiones asevera esa impresión inicial: existe un grupo de características que Jenofonte asocia a pequeñas sociedades virtuosas. Esparta y Persia son, a su modo, naciones reducidas y cerradas, con sistemas que comprenden poca movilidad social y que, a la vez, son exitosos en la reproducción de sus valores tradicionales. La descripción de los ő jenofonteo para la formación de clases gobernantes sólidas y eficientes. Bajo la imagen de sociedades prestigiosas, Jenofonte despliega y pone en acción una serie de elementos teóricos prontos a ser evaluados.

No hay duda que en los marcos iniciales de ambos textos las políticas que regulan

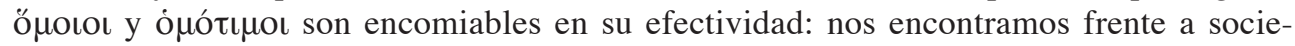
dades estables, sin otóoıs. Pero tanto la Esparta de La constitución de los lacedemonios como la Persia de Ciropedia son transformadas. No ha sido el objeto de nuestro estudio analizar esas mutaciones, pero la patencia de esta situación nos permite arriesgar una interpretación a modo de segunda conclusión. Ambos grupos (y ambas sociedades en su totalidad) sufren rápidamente cambios que alteran su esencia, en lo que parece ser la muestra de un sino aciago. Todo el sistema lacedemonio, incluidos ǒ en el capítulo XIV de La constitución de los lacedemonios, sección en donde Jenofonte critica la realidad espartana contemporánea. Los ó $\mu$ ó $\iota \mu$ oı, en cambio, sufrirán (no sin resistencia) importantes mutaciones en manos de Ciro, cambios que serán imprescindibles para el éxito del proyecto imperial persa: en concreto, los ó $\mu$ ó $\iota \mu$ o dejarán de existir tal y como lo habían hecho tradicionalmente. Acaso en estos profundos quiebres se encuentre una clave para leer la Ciropedia: las políticas emprendidas por Ciro muestran una realidad alternativa al capítulo XIV de La constitución de los lacedemonios, un camino a partir del cual una pequeña sociedad virtuosa puede lograr conformar un imperio a costa de la disolución de alguno de sus rasgos originales. ǒ $\mu$ oı funcionan, independientemente de las realidades históricas que pueden servirles como asiento o excusa, como ejercicios filosóficos para pensar un tipo organización política virtuosa. La constitución de los lacedemonios es, en esta interpretación, una instantánea del pasado perdido que lleva adjunta, en un capítulo, una advertencia de fracaso. La Ciropedia presenta, en cambio, un proceso en pleno movimiento: ya en 2.1.9 el sistema persa se ha alterado de forma en que es imposible volver atrás. A la luz de esto, la revisión conjunta de ǒ $\mu$ oı acceso que permite enriquecer lecturas compartimentadas, mostrando la potencia de las muchas veces subestimada filosofía política de Jenofonte. 


\section{Bibliografía}

AMBLER, W. (2001): Xenophon. The Education of Cyrus, Ithaca: Cornell University Press. BAZIN, H (1885): La République des Lacédémoniens de Xénophon. Étude sur la situation intérieure de Sparte, Paris: E. Leroux.

BIZOS, M. (1971): Xénophon. Cyropédie. Tome I. Libres I-II, Paris: Les Belles Lettres.

BLUDELL, S. (1995): Women in Ancient Greece, Massachusetts: Harvard University Press.

BROWN FERRARIO, S. (2017): "Xenophon and Greek Political Thought", en M. Flower, The Cambridge Companion to Xenophon, Cambridge y New York: Cambridge University Press.

DELEBECQUE, E. (1957): Essai sur la vie de Xénophon, Paris: G. Klincksieck

BOWERSCOCK, G. (1968): Hiero. Agesilaus. Constitution of the Lacedaemonians, London: Loeb.

CAPRA, A. (2010): Donne al parlamento, Roma: Carocci.

CAPRA, A. (2007a), "Stratagemmi comici da Aristofane a Platone. Parte III. Utopia (Repubblica, Donne al Parlamento)", Stratagemmi 4.

CAPRA, A. (2007b): "Stratagemmi comici da Aristofane a Platone. Parte II. L'invettiva (Cavalieri, Gorgia, Repubblica)", Stratagemmi 3.

CAPRA, A. (2007c): "Stratagemmi comici da Aristofane a Platone. Parte I. Il satiro ironico (Simposio, Nuvole e altro)", Stratagemmi 2.

CARTLEDGE, P.A. (1999): “The Socratic's Sparta and Rousseau's”, en S. Hodkinson y A. Powell (eds.): Sparta: New Perspectives, London: Duckworth, pp.311-337.

CASEVITZ, M. (2008): Xénophon, Constitution des Lacédémoniens, Agésilas, Hiéron, Suivi de Pseudo-Xénophon, Constitution des Athéniens, Paris: Les Belles Lettres.

CHRIMES, K.M.T. (1948): The Respublica Lacedaemoniorum adscribed to Xenophon, Manchester: Manchester University Press.

CHRIMES, K.T.M. (1952), Ancient Sparta . A Re-Examination of the Evidence, Manchester: Manchester University Press.

CHRISTESEN, P. (2006): "Xenophon's 'Cyropaedia' and Military Reform in Sparta”, The Journal of Hellenic Studies, No. 126, pp. 47-65.

DANZIG, G. (2009): "Big Boys, Little Boys: Justice and Law in Xenophon's Cyropaedia and Memorabilia", Pólis, No. 26, pp. 242-266

DANZIG, G. (2012): "The Best of the Achaemenids: Benevolence, Self-Interest and the 'Ironic' Reading of Cyropaedia”, en F. Hobden y C. Tuplin, Xenophon: Ethical Principles and Historical Enquiry, Leiden/Boston: Brill.

DE MOURA, J.F. (2002): “A Constituiçao dos Lacedemônios”, en Costa, R. (org), Testemunhos da História, Documentos de História Antiga e Medieval, Vitoria: EDUFES.

DUCAT, J. (2006): Spartan Education: Youth and Society in the Classical Period, Wales: Classical Press of Wales.

DINDORF, L. (1888): Xenophontis Scripta Minora, Leipzig: Teubner.

DUE, B. (1989): The Cyropaedia. Xenophon's Aims and Methods, Aarhus: Aarhus University Press.

FERRARI, F. (2013): Senofonte. Ciropedia, Milano: BUR. 
GEORGES, P. (1994): Barbarian Asia and the Greek Experience, Baltimore: The Johns Hopkins University Press.

GRAY, V. (2007): Xenophon On Government, Cambridge: CUP.

ILLARRAGA, R. (2012): "Utopía ciclópea, utopía de cerdos. Una reconstrucción del pensamiento político de Antístenes a la luz de la sociedad de los cíclopes (FS, I.1014 = SSR, V.A.189) y la ciudad de los cerdos de República, II", en Barbosa, S. y L. Misseri, Estado, cultura y desarrollo: entre la utopía y la crítica, Mar del Plata: EUNMdP.

ILLARRAGA, R. (2017): "El extraño reino de un joven príncipe. Política, educación y justicia en la sociedad persa de la Ciropedia (1,2,2-16)", Quaderni Urbinati di Cultura Classica 116, pp. 81-105.

JOHNSON, D. (2005): "Persians as Centaurs in Xenophon's 'Cyropaedia' “, Transactions of the American Philological Association (1974-), No. 135, pp. 177-207.

KENNELL, N. (2013): "Boys, Girls, Family and the State at Sparta", en Evans Grubbs, J, Parkin, T. y R. Bell (eds), The Oxford Handbook of Childhood and Education in the Classical World, Oxford: OUP, pp. 381-396.

LASCARIDES, V. (2013): History of Early Childhood Education, London: Routledge.

LIPKA, M. (2002): Xenophon's Spartan Constitution, Berlin: de Gruyter.

LUCCIONI, J. (1947): Les idées politiques et sociales de Xénophon, Paris: Ophrys.

LU, H. (2015): Xenophon on Moral Education. Xenophon's Theory of Moral Education, Newcastle Upon Tyne: Cambridge Scholars Publishing.

MACDOWELL, D.M. (1986): Spartan Law, Edimburgh: Scottish Academic Press.

MARCHANT, E. (1920): Xenophontis opera omnia, Oxford: Clarendon Press.

MÁRSICO, C. (2010): Zonas de tensión dialógica. Perspectivas para la didáctica de filosofía antigua, Buenos Aires: Del Zorzal.

MÁRSICO, C., (2013): "Sobre los cerdos. Aspectos de la physis en Antístenes", en C. Mársico y E. Bieda (eds.), Expresar la physis. Conceptualizaciones antiguas sobre la naturaleza, San Martín: UNSAMEdita.

MÁRSICO, C., ILLARRAGA, R. y MARZOCCA, P. (2017): Jenofonte y Pseudo-Jenofonte, Hierón, La constitución de los lacedemonios y La constitución de los atenienses, Buenos Aires: Prometeo/UNQui.

MOORE, J.M. (1983): Aristotle and Xenophon on Democracy and Oligarchy, London: Catto $\&$ Windus, The Hogarth Press.

NADON, C. (2001): Xenophon's Prince. Republic and Empire in the Cyropaedia, California: University of California Press.

OLLIER, F. (1934): Xénophon. La République des Lacédémoniens, Lyon/Paris: F. Alcan.

PRINZ, W. (1911): De Xenophontis cyri Institutione, Göttingen: Dieterich.

RAMÍREZ VIDAL, G. (2005): Jenofonte, La constitución de los atenienses, México: UNAM.

RICHER, N. (1998): Les éphores. Études sur l'histoire et sur l'image de Sparte, Paris: Université de París I, 1998.

RICO GÓMEZ, M. (1989): Jenofonte, La República de los Lacedemonios, Madrid: Centro de Estudios Constitucionales.

ROPER, A (1913): Ancient Eugenics, London: Cliveden. 
SANDRIDGE, N. (2012): Loving Humanity, Learning and Being Honored: The Foundations of Leadership in Xenophon's Education of Cyrus, Washington D.C.: Center for Hellenic Studies Press.

SANSALVADOR, A. (2007): Jenofonte. Ciropedia, Madrid: Gredos.

SCHARR, E. (1919), Xenophon's Staats-und Gesellschaftsideal und seine Zeit, Halle: M. Niemeyer.

TATUM, J. (1989): Xenophon's Imperial Fiction. On the Education of Cyrus, Princeton Princeton University Press.

TIGERSTEDT, E.N. (1965-78): The Legend of Sparta in Classical Antiquity (3 Vols.), Stockholm: Almquist \& Wiskell.

TUPLIN, C. (2002), "Xenophon, Sparta and the Cyropaedia", en Powell, A. y S. Hodkinson (eds.), The Shadow of Sparta, London and New York: Routledge.

VARONA, P. (2009): Jenofonte, Constitución de Esparta, Pseudo-Jenofonte, Constitución de Atenas, Madrid: Cátedra.

WHIDDEN, C. (2007): “The Account of Persia and Cyrus's Persian Education in Xenophon's 'Cyropaedia' “, The Review of Politics, No. 48, pp. 539-567.

WULFF, A. (1884): Quaestiones in Xenophontis de Republica Lacedaemoniorum libello institutae, Münster: Doc. Dissert. 